\title{
Design and Realization of Control System of a Novel Digital Hydraulic 2-DOF Motion Platform
}

\author{
Xiong Xianfeng \\ College of Power Engineering \\ Naval University of Engineering \\ Wuhan, China \\ xianphone@21cn.com
}

\author{
Xing Jifeng \\ College of Power Engineering \\ Naval University of Engineering \\ Wuhan, China \\ jfxing@sohu.com
}

\author{
Peng Likun \\ College of Power Engineering \\ Naval University of Engineering \\ Wuhan, China \\ pelik@163.com
}

\begin{abstract}
Motion platform is widely used to simulate the movement of ship and vehicle, which is of great value for testing of ship and vehicle equipment, motion simulator for training to steer. A novel digital hydraulic 2-DOF motion platform for simulating marine environment is developed, which include incremental stepping motor, four-edge slide spool valve, asymmetric hydraulic cylinder and structure of motion platform, as well as synchronous feedback mechanism. An all-digital control system of the motion platform is designed in accordance with its approximate open-loop control characteristics. The two degrees of freedom of movement of the motion platform can be controlled independently and multiple protection measures are designed to protect the motion platform. It has been proved that the control system not only can control the movement of the platform accurately, but also can provide the anti-interference ability and stable operation system.
\end{abstract}

Keywords-2-DOF motion platform; Digital hydraulic valve; multi-thread;

\section{INTRODUCTION}

As a sports institutions the motion platforms are widely used to simulate movement gesture of the ship, vehicle, aircraft, which has great value in defense and civilian fields, e.g the travel equipment testing, driving simulator and motion simulator for training to steer, etc.

The motion platform of the 6-DOF platform is studied mostly [1].But in many cases, only a few degrees of freedom of movement are needed, which for example, a motion platform of 1, 2, 3, 4 degrees of freedom [2-6]. In aspect of drive technology, servo-motor and servo-hydraulic are the two ways which are used generally. The servo motor driver is usually used in load smaller, control complex and high requires on the oil precision are the insufficient of the servo-hydraulic driver.

In this study, a 2-DOF motion platform is developed with the use of a new type of digital hydraulic technology.

A digital hydraulic integration set, which can be a digital slide spool valve, or a hydraulic cylinder, or a digital hydraulic motor, and or a digital servo valve, is composed of stepping motor and some hydraulic components. This idea of such design and application is existed for a long time [7-8].With the development of stepper motors and microelectronics technology, research in this area is gradually increased in recent years. The American Victory Controls produce a series of digital servo stepper hydraulic cylinder and controller, and provide technical solutions [9]. The German Bosch Rexroth has launched its latest digital hydraulic cylinder in 2003. Zhanghai designed a digital servo hydraulic cylinder based on screw mechanism [10], Wei Xiangyu builds a closed-loop control system digital cylinder with a stepper motor and encoder combination of hydraulic cylinders, and gives the instance for lathes main feed motion of the semi-automatic the turret [11].

This article describes the structural composition and works of the 2 degrees of freedom digital hydraulic motion platform, and the full digital control system is designed according to its approximate open-loop control characteristics. The full digital control system not only can control two degrees of freedom of platform movement independently, but also has advantages of high-accuracy, anti-interference ability, stable and reliable operation.

\section{THE STRUCTURE OF THE 2-DOF MOTION FLATFORM}

An digital hydraulic 2-DOF motion platform is developed by a domestic famous university, which mainly is used to simulate ship 1-DOF or 2-DOF swing movement and is composed of motion platform frame, hydraulic oil source and control system. The 2-DOF motion platform is shown in Figure 1. To the control system, the motion of the roll framework and the pitch framework can be controlled independently.

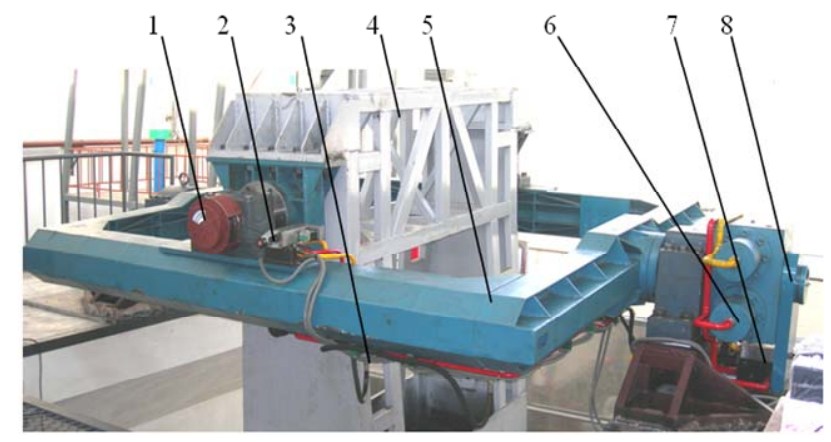

1- pitch feedback mechanism 2- pitch digital hydraulic valve 3- pitch drive cylinder 4-pitch frame 5-roll frame 6-roll swing cylinder 7-roll digital hydraulic valve 8-roll feedback mechanism

Figure.1 A new type of digital hydraulic 2-DOF platform 
The roll mechanism is mainly composed of the roll frame, the hydraulic swing cylinder, the digital hydraulic valve, the feedback timing belt, the roll rotary encoder and bearing support devices and other components. The roll frame is driven by the hydraulic swing cylinder, which is fixed on the foundation. There are two hydraulic cylinders inside of the swing cylinder, the two cylinders can move oppositely, and the roll axis rotation is driven by the rack and pinion mechanism.

Swing cylinder is controlled by the digital hydraulic valve, and the close control of position and velocity is realized in the digital hydraulic valves by the roll angle, which is feedback through the timing directly. For real-time monitoring of the roll axis of the swing angle and the rocking speed, a rotary encoder is connected with the digital hydraulic valve coaxially. The roll bearings are fixed in the foundation, and two short axis connected to the roll framework are supported by the bearings.

The pitch mechanism is mainly composed of the pitch frame, the asymmetric hydraulic cylinders, the digital hydraulic valve, the feedback timing belt, the roll rotary encoder and bearing support devices and other components. The pitch frame is driven by the asymmetric hydraulic cylinder, which rotates the pitch axis through a link mechanism, and is fixed to the frame of the roll framework. The asymmetric hydraulic cylinder is controlled by the digital hydraulic valve, and the closed control of position and velocity is realized in the digital hydraulic valves by the pitch angle, which is feedback through the timing directly. For real-time monitoring of the pitch axis of the swing angle and the rocking speed, a rotary encoder is connected with the digital hydraulic valve coaxially. The bearings supporting two short axis which are connected to the pitch framework are fixed to the frame of the roll framework, and a flange on the minor pitch axis is designed to install the test body.

\section{WORKING PRINCIPLE OF THE MOTION FLATFORM}

The roll mechanism and the pitch mechanism can be controlled independently. The drive body of this 2-DOF motion platform is composed of digital hydraulic valves, swing symmetry hydraulic cylinder for roll frame and single rod the asymmetric cylinder for pitch frame. The institution of the roll mechanism is similar to the pitch mechanism, and their working principles are also basically the same, as shown in Figure 2.

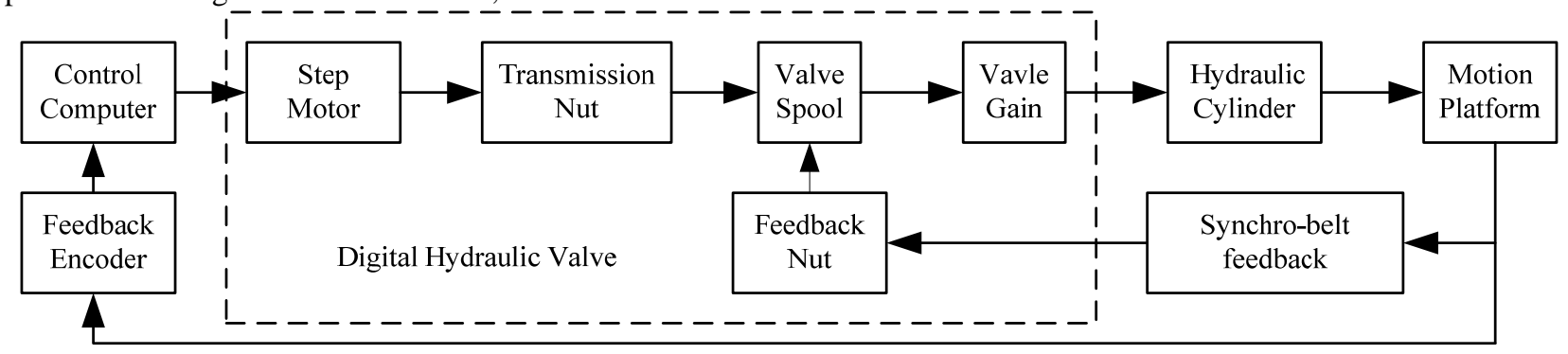

Figure.2 Working principle of the motion platform

Let's take the pitch mechanism for example. When it works, the hydraulic cylinder piston rod telescopic can be controlled by the digital hydraulic valve according swing instruction from the computer. The pitch angle will be transformed into the linear displacement of the slide spool valve by the pitch angle feedback timing belt and the real-time feedback nut. The linear displacement of the slide valve, opposite to the direction of action of the stepping motor, will decrease the opening degree of the valve until it close, so as to achieve the purpose of controlling the swing angle.

Apparently a closed-loop feedback mechanism of mechanical hydraulic servo system is designed on the hardware, and the controller approximation is open loop control because the feedback angle acquiring from the encoder do not really participate in feedback control, but only displays on the PC. So that the control system is only a simple PID controller because the angle, angular velocity, direction of the motion platform can be controlled respectively corresponding the number of pulses, the frequency, direction of the computer.

\section{HardWARE Design OF ThE CONTROL System}

The control system of the new digital hydraulic 2-DOF motion platform is composed of the control computer, motion control card, orthogonal pulse counters, data acquisition card, manual pulse generator and rotary encoders, digital hydraulic valve stepper motors, etc. The institutions schematic of the motion platform is shown in Figure 3.

The control computer, which is responsible for the input and output of the system and data processing, is the core of the entire control system. The motion control card in the control computer is a 2-axis servo/stepper motor control cards, each axis can be controlled independently of each other. The motion control card converses the swing instructions of the motion platform into a certain frequency and number of pulses, which are sent to subdivision driver to drive a stepping motor, so as to control the digital hydraulic valve. By the proportional relation between the pulse number and the swing angle, the real-time actual swing angle of the rolling and pitching can be calculated from the orthogonal pulse signal of the rotary encoder of the roll and pitch axis, which are acquired by the orthogonal pulse counter.

The data acquisition card in the control compute acquires the data of oil temperature, oil pressure, oil level and the filter of the oil source, which are monitored by the control computer whether there is an exception. 


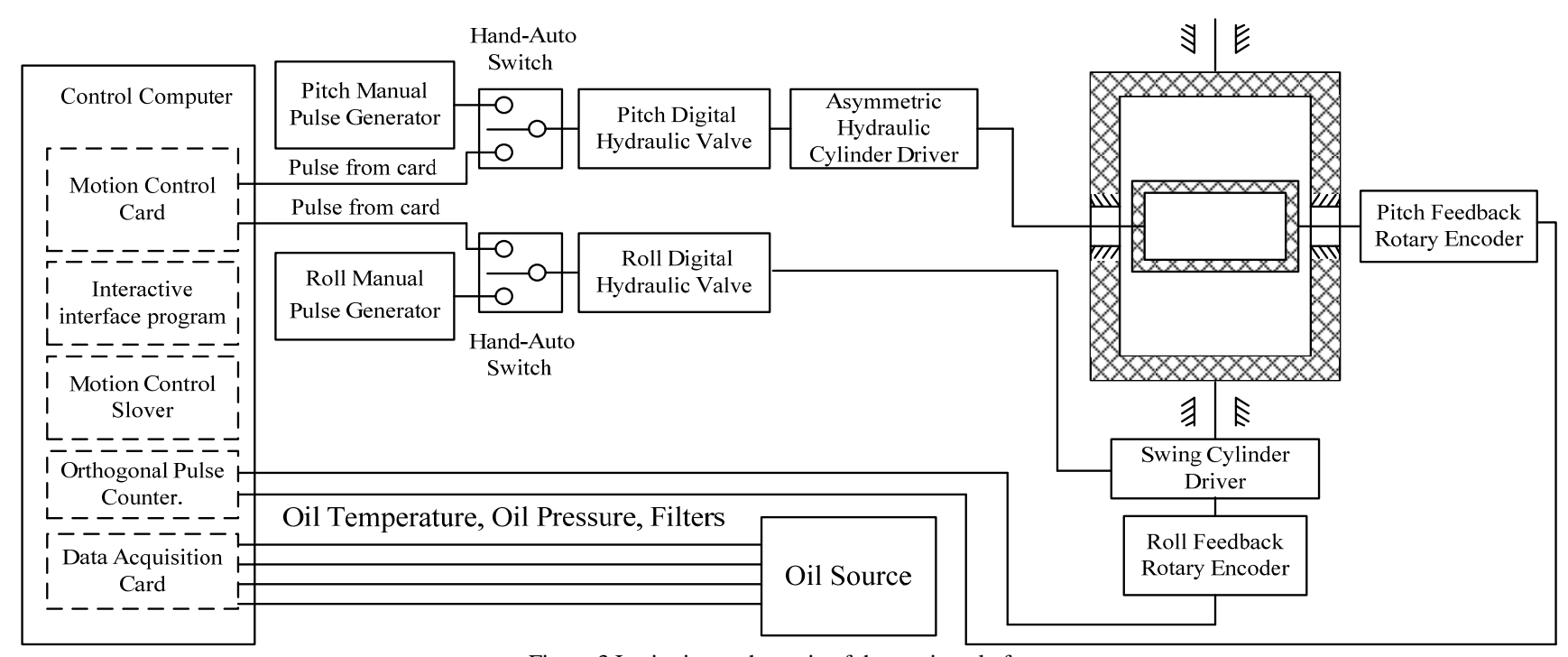

Figure.3 Institutions schematic of the motion platform

Interactive interface program and motion control solver are parts of the software of the control system, which has core function, including setting swing parameters, starting and stopping the platform, displaying information as well as planning and controlling the path based on working parameters.

The control signal of the roll mechanism and the pitch mechanism can be from the computer motion control card and also can be from the manual pulse generator, which can be selected by the hand-auto switch.

In addition, the drive mechanisms of rolling and pitching are different because of the different requirements of swing angle limit and carrying capacity.

Roll drive mechanism applies the swing cylinder driver, which has two hydraulic cylinders and the two cylinders can move opposite, driving the roll axis by the rack and pinion mechanism. Pitching adopts asymmetric hydraulic cylinder driver, which drives the pitching axis through a link mechanism. This system is already a hydraulic servo closed-loop feedback control system because the actual swing angle of the platform acquiring from the rotary encoder has been feedback to the digital hydraulic valve. The actual swing angle only is applied in system monitoring and system protecting, and does not really participate in feedback control, so the system is an openloop control system actually, and the angular velocity, angle and direction of the motion platform are only related to the direction, pulse frequency and quantity.

\section{Programming OF THE CONTROL System}

The control program of the new digital hydraulic control system of 2-DOF motion platform includes interactive interface module and motion control solver module. Interactive interface module runs in the foreground, and the motion control solver module runs in the background.

The interactive interface module is mainly used to start, to reset or to stop the motion platform, to select the roll and pitch control mode (including a separate control and joint control) as well as input their motion parameters (including amplitude, period, and number of cycles), set the data recording interval time and file name, acquire and display the swing angle, oil temperature, oil pressure, filter of the oil source system and limit sensors. In addition, the swing cycle is calculated by motion control solver module, but it also display on the interactive interface.

Motion control solver module plans and calculates the motion path of the platform according to parameters inputting from the interactive interface, and sends the command signal to the implementing mechanism by the motion control card.

The program of the motion control solver module is written in multithreads, including roll automatic thread, roll manual thread, roll back to zero thread, pitch automatic thread, pitch manual thread, pitch back to zero thread, so that the control of the roll mechanism and the pitch mechanism is independently and the system real-time running is improved. In the aspect of program control, roll control and pitch control are independent absolutely, and both of them can be started, reset and stopped alone. The control program flow chart is shown in Figure 4.

In the automatic thread and manual thread, the platform motion path is calculated and planned according to parameters inputting form the interactive interface, and the pulse instructions of every step length, velocity and acceleration are given, which are sent out by the interpolation functions of motion control card, the difference is the manual thread is only responsible for the single-step movement, while the automatic thread is responsible for movement of more than one cycle.

Any control data and current pulse instructions will be cleared when the back to zero thread is effective, and it will detect the direction and speed of the platform motion, calculates and plans platform motion path back to zero position, then the use the interpolation function of the motion control card to give instructions. In the process of the platform back to zero position, the placement, direction and speed of the platform will be detected real-timely, and the number of pulses required is given according to the distance away from the zero 
position, which is proportional to the distance, so as to confirm that the platform can move back to zero position quickly and smoothly without oscillation phenomenon around zero.

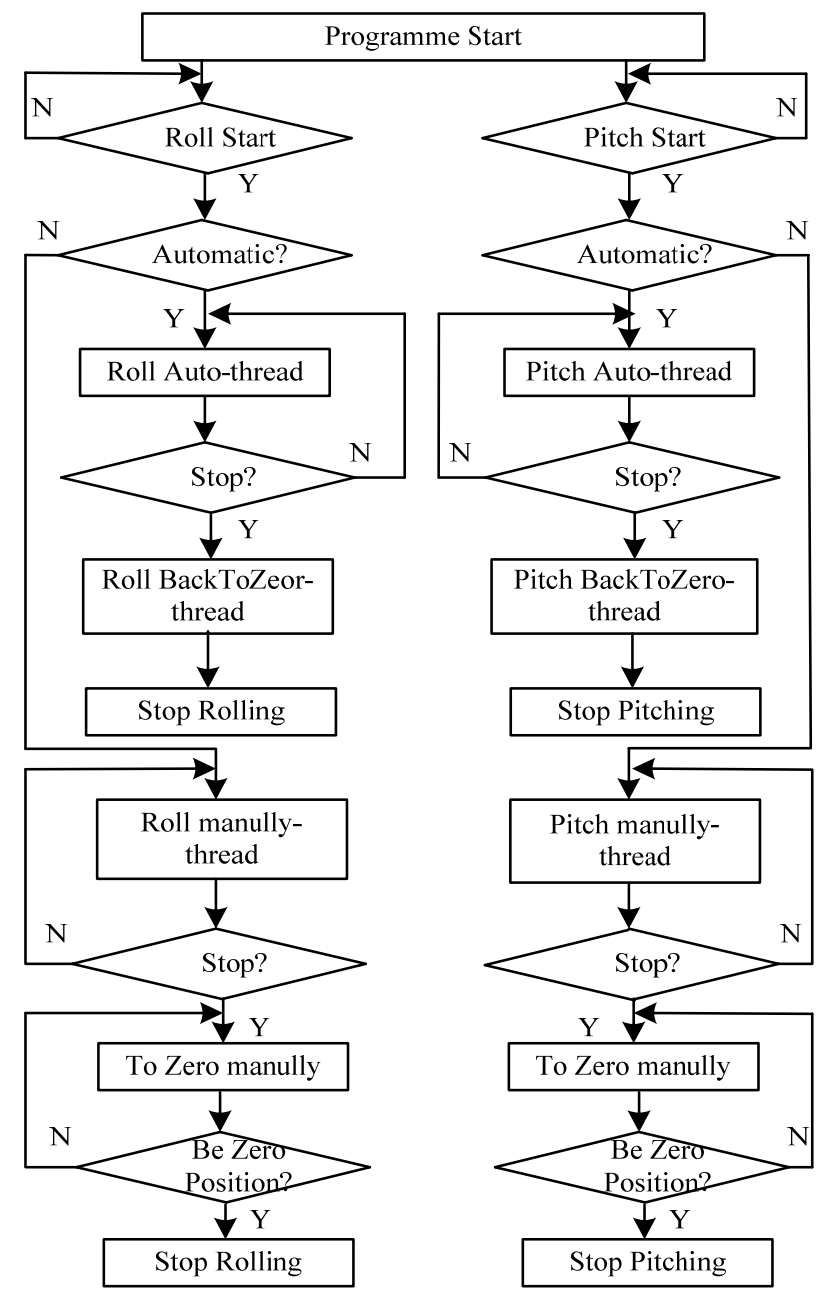

Figure.4 Control program flow chart

In addition, in order to ensure the motion platform running safely and reliably, several security measures are be designed in mechanical design, control hardware and program. In the mechanical design, the roll and pitch mechanism both have mechanical limits, once the platform move to the limit location, the system will be pressure relief automatically and the platform can return to zero itself by gravity.

In the control hardware, the control signal of the roll mechanism and the pitch mechanism can be from the computer motion control card and also can be from the manual pulse generator, which can be selected by the hand-auto switch, in case of emergency, pressing the protection button switch can completely cut off the motion control card and the driver to ensure the safety of the platform. In control program, several limit swing angle values are set, which are less than the mechanical limit angle values. If the roll or pitch feedback angle reaches the set limit values, both the back to zero threads will start automatically, and the automatic thread will re-start and run in accordance with the previously set parameters until both platforms are back to zero position, ensuring the continuity of the motion platform.

\section{CONCLUSION}

A new type of digital hydraulic 2-DOF motion platform is developed based on digital hydraulic technology, which not only is unlike traditional electric or motor servo control system but also is different from a simple closed-loop feedback system with the digital hydraulic cylinder, but a new design of digital mechanical hydraulic servo system based on closed-loop feedback of the swing angle. The control system of the motion platform is fully digital control, and has the advantage in antiinterference ability and stable operation of the system. The multi-threaded program meets real-time demand of the system and high control accuracy. Besides, system-wide safety design features can ensure the safety and reliability of the system.

\section{REFERENCES}

[1] [W. Wang, H. B. Xie and X. Fu, "Optimal method of structural parameters for hydraulic 6-DOF parallel platform based on natural frequency," Chinese Journal of Mechanical Engineering, vol. 42, Mar. 2006, pp. 77-82.

[2] [L. K. Peng, Z. Q. Xiao and J. F. Xing, "Modeling and Experiment Study of a Novel Digital Hydraulic,” Journal of Mechanical Engineering, vol. 47, Feb. 2011, pp. 159-165.

[3] [L. X. Zhang, L. J. Wang and W. He, "Research on simulation for hydraulic platform control system,” Mechatronics, vol. 13, May. 2007, pp. 26-29.

[4] [X. T. Zhao, J. W. Han and J. F. He, "Study on the control strategy of marine 2-DOF platform,” Shipping Engineering, vol. 30, Mar. 2008, pp. 57-59.

[5] [K. T. Zhang, Y. F. Fang and S. Guo, "Design and analyses of a novel 3DOF parallel mechanism,” Journal of Mechanical Engineering, vol. 45, Apr. 2009, pp. 73-76.

[6] [Q. Y. Liu, Y. Li. Liu and Z. Y. Zhou, "Small rotate table with three axes and its control," Instrument Technology and Sensor, vol. 39, Apr. 2002, pp. 9-11.

[7] [X. Y. Wang, J. Cheng, "Dynamic modeling and robust control in task space of 4-DOF parallel electric platform with 4TPS-1PS structure," Journal of Zhejiang University, vol. 43, Aug. 2009, pp. 1492-1496,1548.

[8] [D. Wang, D. Zhou and T. C. Shou, "Research and Development of Digital Hydraulic Valve,” Fluid Power Transmission \& Control, vol. 27, May. 2008, pp. 18-21.

[9] [Y. Z. Xu, D. P. Li and G. X. Chen, "Development and application of digital valve,” Fluid Power Transmission \& Control, vol. 7, Feb. 2010, pp. 5-9.

[10] [H. Zhang, P. C. Gu and Z. X. Pei, "Analysis to the dynamic characteristics of a digital servo cylinder," Journal of Zhejiang University of Technology, vol. 33, Mar. 2005, pp. 295-298,309.

[11] [X. Y. Wei, S. G. Wang and J. Hu, "The design and experiment digital cylinder with inner feedback,” Chinese Hydraulics and Pneumatics, vol. 7, Mar. 2005, pp. 19-22. 